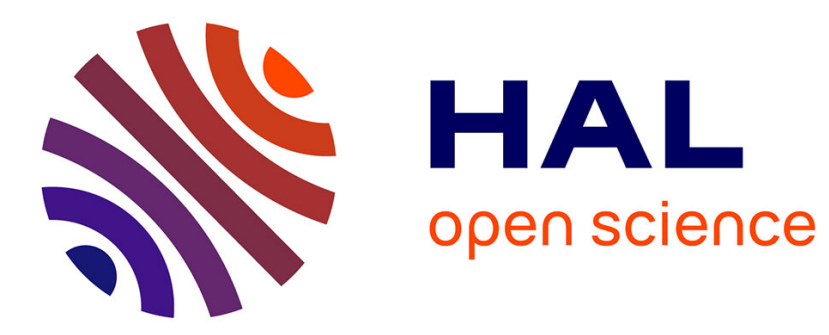

\title{
A classical inversion procedure to obtain the real part of the heavy-ion optical potential
}

R. da Silveira

\section{To cite this version:}

R. da Silveira. A classical inversion procedure to obtain the real part of the heavy-ion optical potential. Journal de Physique Lettres, 1978, 39 (18), pp.311-314. 10.1051/jphyslet:019780039018031100 . jpa00231505

\section{HAL Id: jpa-00231505 https://hal.science/jpa-00231505}

Submitted on 1 Jan 1978

HAL is a multi-disciplinary open access archive for the deposit and dissemination of scientific research documents, whether they are published or not. The documents may come from teaching and research institutions in France or abroad, or from public or private research centers.
L'archive ouverte pluridisciplinaire HAL, est destinée au dépôt et à la diffusion de documents scientifiques de niveau recherche, publiés ou non, émanant des établissements d'enseignement et de recherche français ou étrangers, des laboratoires publics ou privés. 


\title{
LE JOURNAL DE PHYSIQUE-LETTRES
}

Classification

Physics Abstracts

$24.10-25.70$

\section{A Classical InVERSION PROCEDURe TO OBTAIN THE REAL PART OF THE HEAVY-ION OPTICAL POTENTIAL}

\author{
R. DA SILVEIRA
}

Division de Physique Théorique (*), Institut de Physique Nucléaire, 91406 Orsay Cedex, France

(Reçu le 29 juin 1978, accepté le 3 août 1978)

\begin{abstract}
Résumé. - Nous proposons une méthode basée sur une procédure classique d'inversion permettant d'obtenir la partie à longue portée de la partie réelle du potentiel optique entre ions-lourds. Le potentiel est construit à partir de la fonction de déflexion classique que l'on paramétrise à l'aide des données expérimentales de diffusion élastique et inélastique.
\end{abstract}

Abstract. - Using a classical inversion procedure, we propose a method to obtain the long range tail of the real part of the heavy-ion optical potential. The potential is constructed from the knowledge of the classical deflection function which we parametrize from the elastic and inelastic scattering data.

Empirical investigations by Satchler [1] and collaborators [2] show that elastic scattering between heavy-nuclei mainly determines the value of the real part of the optical potential at a rather well defined distance

$$
x \sim D=\eta\left(1+1 / \sin \frac{1}{2} \theta_{1 / 4}\right) / k,
$$

where $\eta=Z_{1} Z_{2} e^{2} / \hbar v$ is the Sommerfeld parameter and $\theta_{1 / 4}$ is the Blair quarter-point angle [3]. Christensen and Winther [4] have shown, using a classical perturbation approach, that $x$ is only slightly smaller than the distance $r_{\mathrm{R}}$ of closest approach for the trajectory leading to the rainbow angle $\theta_{\mathrm{R}}$. The fact that $x$ is close to $r_{\mathrm{R}}$ is not surprising, if one keeps in mind the fact that the angular distribution for elastic scattering and surface reactions is mainly governed by the classical trajectories close to the rainbow situation [5-7]. The purpose of this letter is to present a simple method based on a classical inversion procedure, which enables one to obtain from the experimental elastic and inelastic scattering cross-sections the real part of the optical potential, for distances $r \gtrsim r_{R}$. To this purpose we briefly consider the construction [8] of the potential $V(r)$ from the knowledge of the classical deflection function $\Theta(l)$. Let us define the function $u(r)=r[1-V(r) / E]^{1 / 2}$ for values

(*) Laboratoire associé au C.N.R.S. of $r$ such that $E \geqslant V(r)$. It has been shown [8] that in order to determine $V(r)$ in a unique manner, we must ensure that the inverse function of $u(r)$ exists. It is easy to see that this implies $E>V(r)+\frac{1}{2} r V^{\prime}(r)$. Such a condition cannot be fulfilled for all $r$ satisfying $E \geqslant V(r)$, if $E$ and $V(r)$ are such that orbiting occurs. This means that there is a particular value of $l, l_{1}$, for which the effective potential, $V(r)+\hbar^{2} l^{2} / 2 \mu r^{2}$, exhibits a maximum for $r=r_{1}$, such that

$$
\left[V(r)+\frac{1}{2} r V^{\prime}(r)\right]_{r=r_{1}}=E .
$$

However the domain in which we are interested here is defined by the distances $r$ of closest approach of those trajectories of angular momentum $l \widetilde{S} l_{R}\left(l_{R}\right.$ is the $l$-value of the rainbow trajectory). $l$ and $r$ are related by

$$
E=V(r)+\hbar^{2} l^{2} / 2 \mu r^{2} .
$$

It turns out that $l_{\mathrm{R}}$ is always larger than $l_{1}$. Therefore, for $l>l_{1}$ we have $r>r_{1}$ and $V^{\prime}(r)-\hbar^{2} l^{2} / \mu r^{3}<0$. Eliminating $l$ between this and (1) we obtain the above condition, $E>V(r)+\frac{1}{2} r V^{\prime}(r)$. This ensures the existence of the inverse function of $u(r)$ in the domain of interest. We have the following parametric representation [8] of the function $V(r) ; r=u \exp T(u)$ $V(r)=E\left(1-u^{2} / r^{2}\right)$ and

$$
T(u)=\frac{1}{\pi} \int_{u}^{\infty}\left(b^{2}-u^{2}\right)^{-1 / 2} \Theta(k b) \mathrm{d} b, k b=l .
$$


We now consider the construction of $\Theta(l)$ for values of $l$ such that $l \sim l_{\mathrm{R}}$. We write $\Theta(l)$ in the form

$$
\Theta(l)=\Theta_{\mathrm{n}}(l)+\Theta_{\mathrm{c}}(l), \quad \Theta_{\mathrm{c}}(l)=2 \operatorname{arctg} \frac{\eta}{l} .
$$

At large distances, $r \widetilde{>} \cdot r_{\mathrm{R}}$, the nuclear potential $V_{\mathrm{n}}(r)=V(r)-Z_{1} Z_{2} e^{2} / r$ acts as a perturbation on the Rutherford trajectories and $\Theta_{\mathrm{n}}(l)$, which measures the deviation from pure Coulomb scattering, is very small (a few degrees for energies well above the Coulomb barrier). Substituting (3) in (2), we obtain

$$
\begin{gathered}
T(u)=T_{\mathrm{n}}(u)+T_{\mathrm{c}}(u), \\
T_{\mathrm{c}}(u)=\ln \left\{\frac{\eta}{k u}+\left[1+\left(\frac{\eta}{k u}\right)^{2}\right]^{1 / 2}\right\} .
\end{gathered}
$$

It is easy to see that $u \exp T_{\mathrm{c}}(u)$ is the unperturbed distance of closest approach $r_{0}$ (i.e., that determined by the pure Coulomb field). Therefore $\left|T_{\mathrm{n}}(u)\right| \ll 1$ and we obtain to first order

$$
\begin{gathered}
r=r_{0}\left(1+T_{\mathrm{n}}(u)\right) \\
\text { and } V(r)=Z_{1} Z_{2} e^{2} / r_{0}+2 E \frac{u^{2}}{r_{0}^{2}} T_{\mathrm{n}}(u) .
\end{gathered}
$$

To go further and calculate $T_{\mathrm{n}}(u)$, we parametrize $\Theta_{\mathrm{n}}(l)$, which cannot be constructed from the experiment for all values of $l$. The existence of infinite interpolations shows that the final result is not strictly unique [9]. Nevertheless we believe that the procedure should give an answer very close to the truth. Earlier investigations [10] on the dependence of the rainbow angle on different potential shapes, suggest the very simple form

$$
\Theta_{\mathrm{n}}(l)=\beta \exp \left[\left(l_{\mathrm{R}}-l\right) / \delta\right], \quad l \sim l_{\mathrm{R}} .
$$

The two conditions, $\Theta\left(l_{\mathrm{R}}\right)=\theta_{\mathrm{R}}$ and $\Theta^{\prime}\left(l_{\mathrm{R}}\right)=0$, determine $\beta$ and $\delta$ in terms of two physical parameters $\theta_{\mathrm{R}}$ and $l_{\mathrm{R}} ; \beta=\theta_{\mathrm{R}}-\Theta_{\mathrm{c}}\left(l_{\mathrm{R}}\right)$ and $\delta=\beta / \Theta_{\mathrm{c}}^{\prime}\left(l_{\mathrm{R}}\right)$. If one substitutes (5) in (2), the resulting integral is obtained in a closed analytical form. For $k u / \delta \gg 1$ it gives

$$
T_{\mathrm{n}}(u)=\beta(\delta / 2 \pi k u)^{1 / 2} \exp \left[k\left(b_{\mathrm{R}}-u\right) / \delta\right] .
$$

We now proceed to extract $\theta_{\mathrm{R}}$, the angle for the grazing trajectory, and the corresponding angular momentum $l_{R}$, from the experimental data. In order to obtain $\theta_{R}$ we proceed as in ref. [10] by taking advantage of the fact that quasi-elastic processes, when induced by nuclear forces (nuclear excitation and neutron surface transfer reactions), give rise to a scattering crosssection which exhibits a rather pronounced maximum at the corresponding angle of grazing trajectory. We shall use here the inelastic scattering data in order to obtain $\theta_{\mathbf{R}}$. For this we take the angle corresponding to the maximum of the cross-section in the large angle region which, when well separated, contains small admixtures from Coulomb excitation (Fig. 1). The

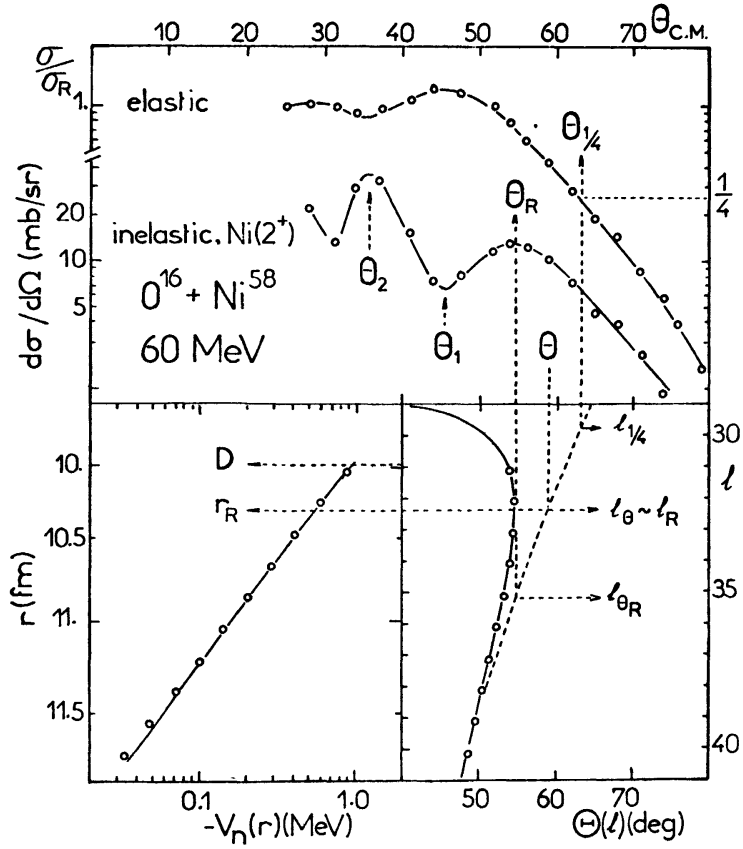

FIG. 1. - Upper part : Experimental data of elastic and inelastic scattering of $\mathrm{O}^{16}$ on $\mathrm{Ni}^{58}$ at $E_{\mathrm{L}}=60 \mathrm{MeV} . \theta_{\mathrm{R}}$ is the experimental rainbow angle (see the text). The angles $\theta_{1}, \theta_{2}$ and $\theta_{1 / 4}$ serve for the determination of $l_{\mathrm{R}}$. Curves are drawn to guide the eye. Lower-right part : The classical deflection function calculated, with the WoodsSaxon potential which fits the data (full curve) and the parametrized form, expression (5) (open circles). The broken curve represents the pure Coulomb result. Lower-left part : Full curve represents the Woods-Saxon potential and open circles, the values of the calculated potential. $D$ and $r_{\mathrm{R}}$ are respectively the strong absorption radius and the distance of closest approach of the rainbow trajectory. Note that the $r$-scale on the left is not linear although the corresponding $l$-scale on the right, is. $l$ and $r$ are related by the expression (1).

way we propose to extract $l_{\mathrm{R}}$, although essentially empirical, appears to be quite accurate. We first determine a lower bound of $l_{\mathrm{R}}$. For $l<l_{\mathrm{R}}, \Theta(l)$ decreases rapidly (even approaching $-\infty$, if orbiting occurs) within a few units of $l$. This region of $\Theta(l)$ is commonly called the nuclear branch. The interference between this branch and the Coulomb one $\left(l \gg l_{\mathrm{R}}\right)$, gives rise to the small angle oscillations of elastic and inelastic scattering [5-7]. We obtain an accurate estimate of the l-location of the nuclear branch, $l=l_{\mathrm{a}}$, by using the method developed in ref. [11]. Taking the first minimum $\theta_{1}$ and the first maximum $\theta_{2}$ (starting from $\theta_{R}$ ) we have as a lower bound of $l_{R}$ (Fig. 1)

$$
l_{\mathrm{a}}=\left(2 \eta \ln \frac{\sin \frac{1}{2} \theta_{1}}{\sin \frac{1}{2} \theta_{2}}-\pi\right) /\left(\theta_{1}-\theta_{2}\right) .
$$

Therefore we have [11] $l_{\mathrm{a}} \sim l_{1 / 4}=\eta \operatorname{cotg} \frac{1}{2} \theta_{1 / 4}$. In order to obtain $l_{\mathrm{a}}$ via $l_{1 / 4}$ we need only know one angle $\left(\theta_{1 / 4}\right)$ in contrast to the $l_{\mathrm{a}}$ given by (6) for which one needs to know two angles $\left(\theta_{1}, \theta_{2}\right)$. The first method is then particularly useful if, at a given energy, only the inelastic data are available. Such a situation will be 
examined below. An upper bound of $l_{R}$ can be obtained by calculating the $l$-value $l_{\theta_{\mathrm{R}}}$ of the Rutherford trajectory which is deflected through the angle $\theta_{\mathbf{R}}$. We now have $l_{\mathrm{a}}<l_{\mathrm{R}}<l_{\theta_{\mathrm{R}}}$. It appears that an accurate estimate of $l_{R}$ is given by $l_{R}=\eta \operatorname{cotg} \frac{1}{2} \theta$, with $\theta=\left(\theta_{\mathrm{R}}+\theta_{1 / 4}\right) / 2 \operatorname{or} \theta=\left(\theta_{\mathrm{R}}+\theta_{\mathrm{a}}\right) / 2, \theta_{\mathrm{a}}=2 \operatorname{arctg} \frac{\eta}{l_{\mathrm{a}}}$.

We now consider the application of the method to the illustrative example given in figure 1. In the upper part of the figure are plotted the experimental data [12] of elastic and inelastic $\left(\mathrm{Ni}, 2^{+}\right)$scattering of $\mathrm{O}^{16}$ on $\mathrm{Ni}^{58}$ at $E=60 \mathrm{MeV}$. From these data, we have $\theta_{1} \sim 45.5^{\circ}, \theta_{2} \sim 36^{\circ}, \theta_{\mathrm{R}} \sim 54.5^{\circ}$ and $\theta_{1 / 4} \sim 63^{\circ}$. From (6) we obtain $l_{\mathrm{a}}=30.3$ which gives $\theta=58.26^{\circ}$ and $l_{\mathrm{R}}=32.7$, and from $\theta_{1 / 4}$ we have $\theta=58.75^{\circ}$ and $l_{R}=32.4$. In the lower-right part of the same figure the classical deflection function is plotted - full curve - calculated with the real part of a WoodsSaxon potential [12] which fits the data (this potential will be called the experimental potential). The open circles represent the values of the parametrized deflection function. In the lower-left part wa compare the calculated nuclear potential - open circles - with the experimental one - full curve. Figure 2 shows the result of the same calculation for some other situations. The angles $\theta_{\mathrm{R}}$ and $\theta_{1 / 4}\left(\right.$ or $\theta_{1}$ and $\theta_{2}$ ) as well as the $l_{\mathrm{R}}$ values are given in table $I$. Full curves represent the Woods-Saxon experimental potentials (parameters in table I) and the broken curves represent the result of our calculations. For $\mathrm{C}^{12}+\mathrm{Pb}^{208}$ only inelastic data are available at $98 \mathrm{MeV}$. In this case $\theta$ has been obtained via $\theta_{\mathrm{a}}$ after calculating $l_{\mathrm{a}}$ from (6). The experimental potential is obtained [2] from the elastic data at $96 \mathrm{MeV}$. In all the cases $V(r)$ is calculated for values of $r$ greater than that corresponding to $l \sim 0.95 l_{\mathrm{R}}$, which includes the point $x=D$ indicated by the arrows. Before we discuss these results, it is interesting to compare the $l_{R}$ values obtained by the present method, which although empirical is model independent, with those obtained from a model dependent calculation. It has been shown [4] that for

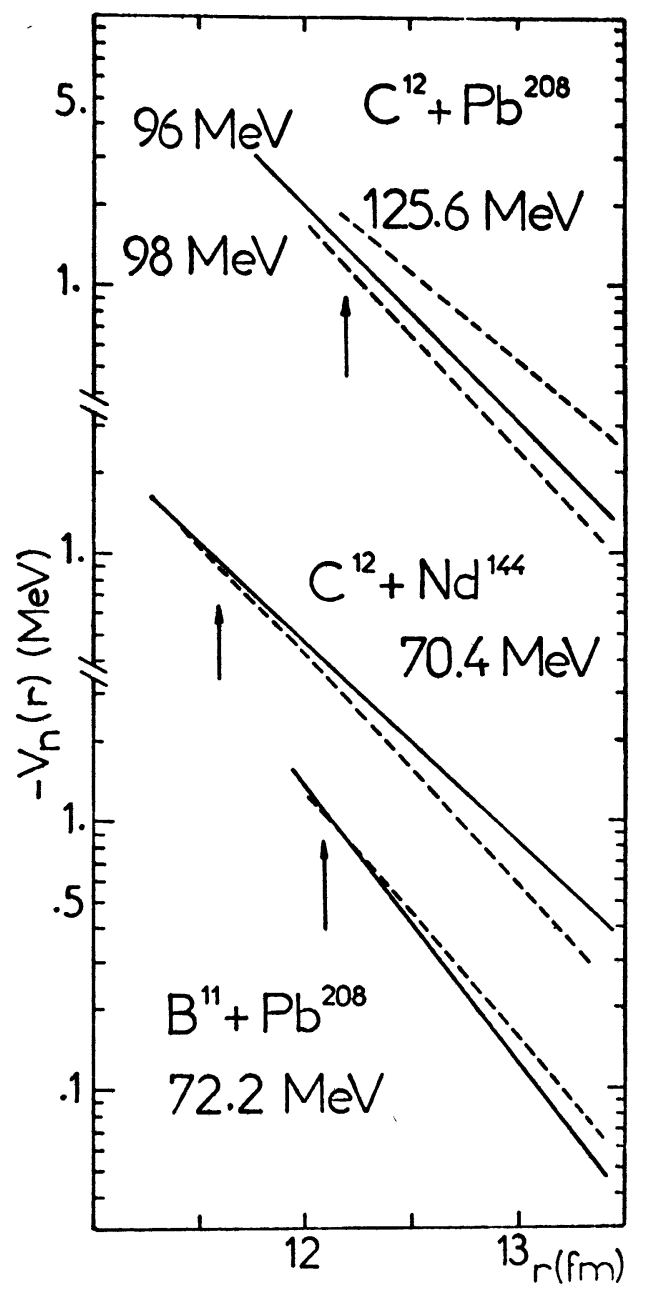

Fig. 2. - Comparison of the calculated potential (broken curves) with those potentials (Woods-Saxon) fitting the data (full curves). The arrows indicate the distance at which different potentials, giving equally fits to the data, cross each other.

an exponential tail shape potential of diffuseness $\alpha$, $l_{\mathrm{R}}$ closely approximates

$$
l_{\mathrm{R}}+\frac{1}{2}=\eta \operatorname{cotg}\left(\frac{1}{2} \theta_{\mathrm{R}}+\frac{\sin ^{2} \frac{1}{2} \theta_{\mathrm{R}}}{\cos \frac{1}{2} \theta_{\mathrm{R}}} \frac{2 \alpha E}{Z_{1} Z_{2} e^{2}}\right) .
$$

\section{TABLE I}

\begin{tabular}{cccccccccccc}
\multicolumn{10}{c}{$\begin{array}{c}\theta_{1 / 4} \\
\text { or }\end{array}$} \\
Ref. & Projetile & Target & $E$ & $\theta_{\mathbf{R}}$ & $\theta_{1}-\theta_{2}$ & $l_{\mathbf{R}}$ & $l_{\mathbf{R}}^{\prime}$ & $V_{\mathbf{O}}$ & $r_{\mathbf{0}}$ & $\alpha$ & $\begin{array}{c}\text { level } \\
-\end{array}$ \\
\hline 13 & - & - & - & - & - & - & - & - & - & - & - \\
14 & $\mathrm{~B}^{11}$ & $\mathrm{~Pb}^{208}$ & 72.2 & 60 & 67 & 40.7 & 40.5 & 21.8 & 1.334 & 0.416 & $\mathrm{~Pb}\left(3^{-}\right)$ \\
15 & & $\mathrm{Nd}^{144}$ & 70.4 & 57 & 65 & 39.7 & 39.1 & 20 & 1.315 & 0.562 & $\mathrm{Nd}\left(4^{+}\right)$ \\
16 & & $\mathrm{~Pb}^{208}$ & 98 & 49 & $42-35$ & 55.6 & 55.1 & 23 & 1.31 & 0.52 & $\mathrm{~Pb}\left(3^{-}\right)$ \\
12 & $\mathrm{O}^{16}$ & $\mathrm{~Pb}^{208}$ & 125.6 & 33 & 37.5 & 75.4 & 76.2 & & & & $\mathrm{~Pb}+\mathrm{C}$ \\
& & $\mathrm{Ni}^{58}$ & 60 & 54.5 & 63 & 32.4 & 31.9 & 25.3 & 1.3 & 0.533 & $\mathrm{Ni}\left(2^{+}\right)$
\end{tabular}

The energy $E(\mathrm{Lab}, \mathrm{MeV})$ the rainbow angle $\theta_{\mathrm{R}}$ and the angles $\theta_{1 / 4}$ or $\left(\theta_{1}, \theta_{2}\right)$ (in degrees) which serve to determine $l_{\mathrm{R}}, l_{\mathrm{R}}^{\prime}$ in the $l_{\text {-value }}$ obtained from expression (7). $V_{0}(\mathrm{MeV}), r_{0}(\mathrm{fm}), \alpha(\mathrm{fm})$ are the parameters of the Woods-Saxon potential which fits the data. The column level, indicates the inelastic angular distribution from which $\theta_{R}, \theta_{1}$ and $\theta_{2}$ has been extracted. 
We list in the column $l_{R}^{\prime}$ of table I the $l_{R}$ values obtained from this expression, evaluated with the diffuseness $\alpha$ of the experimental potential. The results obtained with (7) and those obtained by the present method, differ at most by about $1.5 \%$. Returning to figures 1 and 2 , we see that the calculated potentials have an almost exponential tail and that they are a rather good approximation of the experimental potentials in the vicinity of $x=D$. This is also the region where the result is less dependent on the particular choice of the parametric form of $\Theta(l)$. We close by recalling that Satchler [1] has shown that the real part of those potentials which give equally good fits to experimental data, cross each other approximately at the same point, near $x=D$. Since the potentials obtained here cross the experimental ones at approximately the same distance, it can be expected that they will possess a similar ability to reproduce the data.

The author wishes to acknowledge Dr. S. Klarsfeld for fruitful discussions.

\section{References}

[1] Satchler, G. R., Proc. International Conf. on reactions between complex nuclei, Nashville, (North Holland) 1974, vol. 2, p. 171.

[2] Ball, J. B., Fulmer, C. B., Gross, E. E., Halbert, M. L., Hensley, D. C., Ludemann, C. A., Saltmarsh, M. J. and SATCHLER, G, R., Nucl. Phys. A 252 (1975) 208.

[3] Blair, J. S., Phys. Rev. 95 (1954) 1218.

[4] Christensen, P. R. and Winther, Aa., Phys. Lett. 65B (1976) 19.

[5] Broglia, R. A., Landowne, S. and Winther, Aa., Phys. Lett. 42 (1972) 293.

[6] Malfliet, R. A., Landowne, S. and Rostokin, V., Phys. Lett. 44B (1973) 238.

[7] DA SilveIra, R., Phys. Lett. 45B (1973) 211.

[8] Miller, W. H., J. Chem. Phys. 51 (1969) 3631.

For a general view of the W.K.B. solution of inverse problems, see Buck, U., Rev. Mod: Phys. 46 (1974) 369 and also Wheeler, J. A., Phys. Rev. 99 (1955) 630, where it is suggested that this solution is possible.
[9] Sabatier, P. C., J. Math. Phys. 13 (1972) 675.

[10] DA Silveira, R., Phys. Lett. 50B (1974) 237.

[11] DA Silveira, R., Nucl. Phys. A 272 (1976) 208. The angles $\theta_{1}$ and $\theta_{2}$ can also be extracted from the elastic data see this reference.

[12] Christensen, P. R., Chernov, I., Gross, E. E., Stokstad, R. and VidebaCK, F., Nucl. Phys. A 207 (1973) 433.

[13] Ford Jr., J. L. C., Toth, K.S., Hensley, D. C., GaedKe, R. M., Plley, P. J. and Thornton, S. T., Phys. Rev. C 8 (1973) 1912.

[14] Hillis, D. L., Gross, E. E., Hensley, D. C., Rickertsen, L. D., Bingham, C. R., Scott, A. and BAKer, F. T., Phys. Rev. Lett. 36 (1976) 304.

[15] Satchler, G. R., Ford Jr., J. L. C., Toth, K. S., HensLEY, D. C. and Gross, E. E., Phys. Lett. 60B (1975) 43.

[16] Wang, K. H. and MC InTyre, J. A., Proc. 3rd Conf. on reactions between complex nuclei, Asilomar (1963) p. 31. 